managing group dynamics and how to facilitate supervision remotely using video conferencing. Staff from different disciplines attended including therapists, nurses, social workers and counsellors. Bi-monthly meetings were arranged to support the group.

It has led to increasing the availability of supervision with seven of those who attended training now offering regular group supervision and drop-in sessions to our inpatient staff, living well team and non-clinical staff who have patient contact. Initial findings from surveying those who offered and received supervision suggest greater inter-disciplinary work and improved staff satisfaction. We discuss the results in terms of the resilience of our workforce in the context of pandemic, potential financial benefits and further opportunities and challenges.

\section{P-191 CARING FOR OUR PEOPLE, A PROGRAMME FOR RESTORATIVE SUPERVISION}

Jennifer Latchford, Julie Hallatt, Abigail Davison. St Leonard's Hospice, York, UK

10.1136/spcare-2021-Hospice.207

A programme of Restorative Supervision was introduced in May 2021 in response to the pressures observed on staff working in a hospice setting during a pandemic. Restorative Supervision aimed to bridge the gap between the existing staff support framework and provision for staff welfare. The aim was to maintain and restore collective wellbeing from the roots up by supporting the individual. The offer was directed to all patient-facing staff.

We researched different models including 'The Restorative Resilience Model of Supervision' developed by Dr Sonia Wallbank. The family support team including our spiritual care lead, bereavement lead and social workers were then trained to deliver the programme.

One of the staff groups experiencing significant stress during the pandemic were the Housekeeping Team. Their workload had increased significantly as they worked with the increasing pressure to maintain the safety of everyone else in the building. This put them under great physical and emotional stress. We identified housekeeping as our pilot group and began the roll out. We discussed the aim of the programme with the Head of the Department and ensured everyone was given a protected hour to attend a restorative session. We explained that the aim was to enable staff to protect their capacity to think and care for themselves and others.

In order to measure the quality and outcomes of the sessions we used two forms; a wellbeing questionnaire and a feedback form. The evidence so far suggests that staff welcomed the opportunity to talk in confidence and have felt 'listened to' in a safe and confidential space. We are now moving to the inpatient unit staff including nursing and care teams and will continue to collect evidence.

\section{P-192 THE DEVELOPMENT OF 'WALK AND TALK' SUPERVISION FOR CLINICAL STAFF WITHIN A HOSPICE WORKING THROUGHOUT THE PANDEMIC}

Tracey Addy, Seamus Nash. The Kirkwood, Huddersfield, UK
The Kirkwood multidisciplinary team (MDT) have embraced clinical supervision across all clinical departments and thus the clinical teams have felt supported and sustained whilst working through the pandemic.

The Kirkwood counselling team have provided supervision to MDT colleagues both in mixed groups and individually to staff. The concept of 'Walk and Talk' supervision was expressly developed as it was identified that sections of clinical staff felt increasingly isolated as they continued working remotely, from home. Staff who attended work on-site also felt equally isolated from colleagues as they felt a loss of and lack of sustaining contact with colleagues. All staff had to change their usual manner of working with attendant discomfort of routines.

Initially supervision was facilitated via video calls, however, not all staff had camera-enabled computers and this loss of visual contact was a huge barrier to engagement at depth. As this proved unsustainable, the idea of integrating nature focused, walking supervision took shape.

Counsellors took the NHS 'Steps to Mental Wellbeing' and adapted these to include:

- Connecting with others.

- A focus on being physically active.

- A commitment to learn new skills.

- A focus on gratitude.

- Integrating mindfulness and paying attention the present moment.

By walking alongside colleagues, the supervisor created an environment where threat was minimised which enhanced maximum engagement. Colleagues felt that this activity resulted in them feeling less exposed and reported that they felt less self-conscious reporting material. The message on the NHS website was re-enforced within the supervision space by direct human presence and contact so that colleagues were not required to 'rely on technology or social media alone to build relationships'.

\section{P-193 'YOU'RE ON MUTE!': SCHWARTZ IN A GLOBAL PANDEMIC}

Sarah Dowd, Clair Sadler, Vanessa Hill, Lizzie Lee, Laura Yalley-Ogunro. Princess Alice Hospice, Esher, UK

\subsection{6/spcare-2021-Hospice.209}

Background Hospice staff encounter distress, physical and psychological pain every day (Pauly, Varcoe, Storch, 2012). Schwartz Centre Rounds (SCR) promote compassionate care and its link to patient experience (Point of Care Foundation). But who cares for the carer? SCR provide space for reflection, and shared experiences (Cullen, 2012) and during COVID-19 this is more vital than ever (Newcomb, 2021); our hospice Schwartz Steering Group was keen to continue SCR online to support colleagues. 'Reflection helps manage our responses and lives can be enhanced by cultivating compassion in our practice' (Sansó, Galiana, Oliver et al., 2015).

Aims

- Launch and sustain virtual monthly SCR for staff/volunteers to reflect and connect.

- Obtain feedback on virtual format through survey.

- Identify future SCR topics.

- Help decision making with SCR future facilitation. 